\title{
Inflammatory Myofibroblastic Tumor of Lung - Computed Tomographic Features in 20 Patients
}

\author{
Ghimire $\mathbf{P}^{1}$, Zhu L $\mathrm{L}^{2}$, Wu G \\ ${ }^{1}$ Department of Radiology, Nepalgunj Medical College and Teaching Hospital Kohalpur, \\ Banke, Nepal, ${ }^{2}$ Department of MRI, Zhongnan Hospital of Wuhan University, Wuhan, \\ Hubei, 430071, PR China
}

\begin{abstract}
Aim: To study the salient characteristic computed tomographic findings of inflammatory myofibroblastic tumor of lung. Materials and methods: We retrospectively reviewed the CT examinations of twenty histopathologically confirmed cases of inflammatory myofibroblastic tumor of lung and analyzed the involvement, predominant location, pattern of presentation, shape, edge, pattern and degree of enhancement and any atypical findings in those cases. Results: Fourteen cases presented as pulmonary nodules among which twelve as solitary while two cases as multiple pulmonary nodules. Six cases presented as masses. The location was in the parenchyma of the lung among all cases except two masses that were predominantly mediastinal and endobronchial respectively. All nodules demonstrated mild enhancement except one nodule showed moderate and another one marked enhancement. Four masses demonstrated mild enhancement whearas one showed moderate and another marked enhancement. Pleural surface abutting was noticed in one nodule and two masses. Stippled calcification was present in one mass while necrosis was noticed in two other cases that presented as mass. Mass were associated with consolidation in one and as cavity in another case. Conclusion: Although diagnosis of inflammatory myofibroblastic tumor of lung cannot be confirmed radiologically, certain features as presence of nodules or masses that enhance mildly in a patient with equivocal clinical and radiological presentation warrants its inclusion in the differential diagnosis.
\end{abstract}

Keywords: Computed tomography, Inflammatory, Myofibroblastic

\section{Introduction}

Inflammatory pseudotumor of the lung is a benign entity which mimics other benign and malignant lesions of the lung. With etiology unknown, these tumors are rare in occurrence though they are the most common cause of solitary nodule or mass in children and adolescent with no any sex predilection. They are usually an incidental finding in majority of the cases while some present with clinical symptoms of cough, chest pain, fever and dyspnea. They are usually peripheral in location in the parenchyma with very few cases presenting as mediastinal or endobronchial or endotracheal masses. The purpose of our study is to identify computed tomographic features of inflammatory myofibroblastic pseudotumors in 20 histopathologically confirmed cases which are useful in characterization of the lesions.

Correspondence to: Dr. Prasanna Ghimire, Department of Radiology, Nepalgunj Medical College and Teaching Hospital Kohalpur, Banke, Nepal

E-mail- drprasannaghimire@ gmail.com 


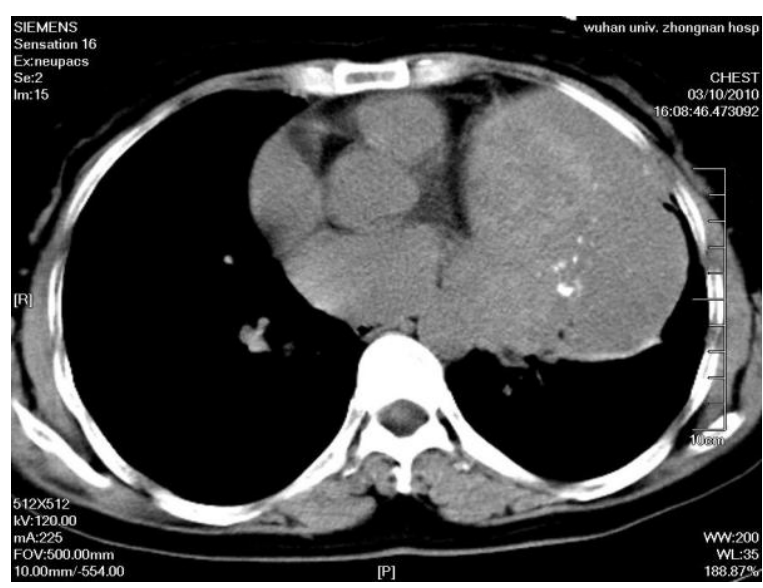

Fig 1a: Non-contrast enhanced CT scan demonstrates a lobulated homogeneous mass in the lower lobe of the left lung. Lesion is abutting the chest wall and demonstrates few calcifications.

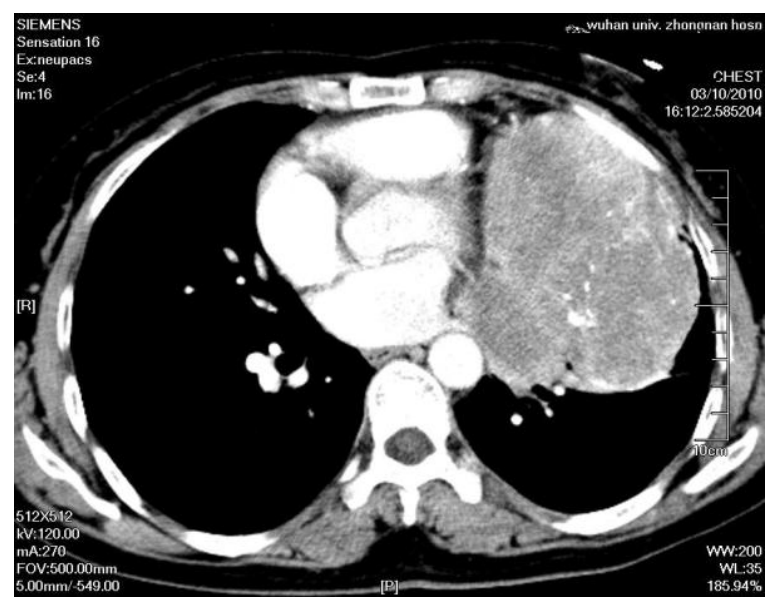

Fig 1b: Contrast enhanced CT scan demonstrates heterogeneous enhancement of the lesion.

\section{Materials and methods}

A retrospective review of pathological database from January 2004 through 2009 revealed 33 cases diagnosed with inflammatory pseudotumor of the lung. Of these cases, 23 cases had undergone chest CT at our department. We excluded three cases which did not have both the plain and contrast CT images. Among the twenty patients, 13 were male and 7 were female with a mean age of $38.9 \pm$ SD 11.66 years (range 16-61years). Fourteen patients were asymptomatic while three had presented with chest pain, one with cough, one case with fever and weight loss and another case with hemoptysis.

\section{Image Acquisition}

CT scan was performed using Siemens Sensation 16-slice CT scanner, using 16x1.5 $\mathrm{mm}$ collimation and slice thickness of $3 \mathrm{~mm}$ from clavicular heads through both costophrenic angles. Contrast enhanced scans were taken 30 seconds after injecting $80 \mathrm{ml}$ of Isovue 300 via power injector at the rate of $2.5 \mathrm{ml} / \mathrm{sec}$.

\section{Image Analysis}

CT images were brought to the local PACS workstation and were analyzed for the involvement as - site ( right upper, middle, lower lobe or left upper, lower lobe, lingual or multifocal), predominant location (parenchymal, mediastinal or endobronchial or combination), pattern of presentation (solitary pulmonary nodule, multiple pulmonary nodule, mass, consolidation, cavity), size (maximum dimension), shape (oval, round, lobular, irregular), edge (well defined or poorly defined), attenuation (homogeneous or heterogeneous), degree of enhancement (mild, moderate or marked) and any atypical findings (calcification, abutting pleura, necrosis, etc).

\section{Results}

1. Twelve cases presented as solitary pulmonary nodule with lesion location in the parenchyma of the lungs. Two cases presented as multiple pulmonary nodules. The mean maximum dimension of the nodules was $2.9 \mathrm{~cm}$. Shape of the nodule was round in all cases except in one case which was oval. All nodules were with well circumscribed margins and homogeneous attenuation. All nodules showed mild enhancement except one which showed moderate and another one marked enhancement. One case showed nodule abutting the pleural surface. 


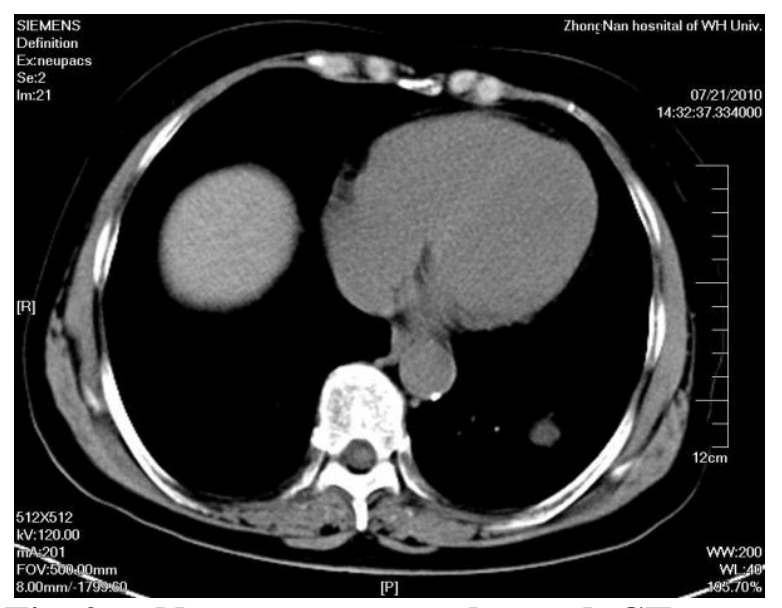

Fig 2a: Non- contrast enhanced CT scan demonstrates a homogeneous nodule in the left lower lobe.

2. Six cases presented as masses. One mass presented with consolidation while another with cavity. All cases had predominant lesion in the parenchyma except two cases which had the predominant lesion in the mediastinum and another endobronchial. The mediastinal lesion had extended to the parenchyma. The shape of the masses was round (1 case), lobulated (3 cases) and irregular (1 case). The attenuation was heterogeneous in five of them and homogeneous in one. All masses showed mild contrast enhancement except one which showed moderate and another marked enhancement. All but two of the masses were abutting the pleural surface. The masses ranged from $4.9 \mathrm{~cm}$ to $11.2 \mathrm{~cm}$. One mass had internal calcification, while two masses had central necrosis.

\section{Discussion}

Inflammatory pseudotumors are rare quasineoplastic lesions with lungs being the most common site of involvement. They have propensity to mimic malignant lesions both clinically and radiologically thus posing diagnostic challenges. The overall incidence of IMT of the lung is reported to be $0.04 \%$ $1 \%$ of all tumors of the lung. ${ }^{1}$ These tumors are seen in all age groups but are the most common primary tumor like lesion of the lung in children and adolescents. The mean

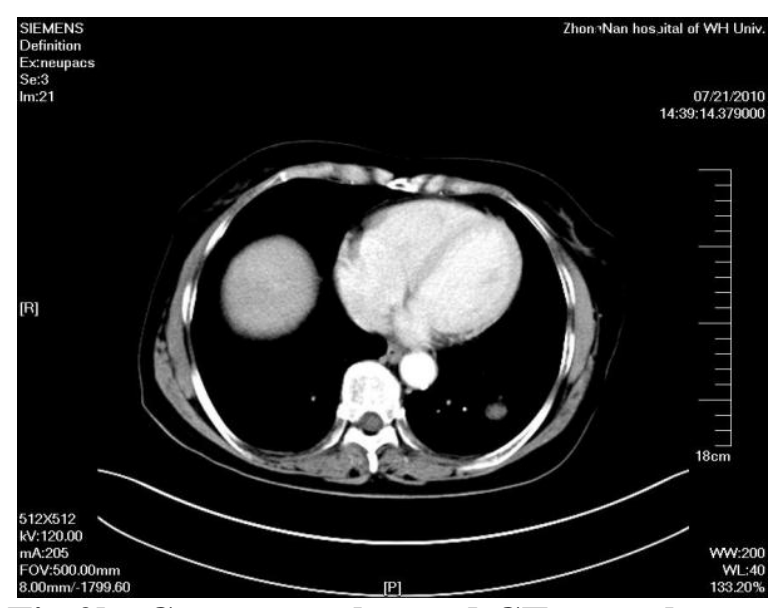

Fig 2b: Contrast enhanced CT scan shows mild enhancement of the nodule.

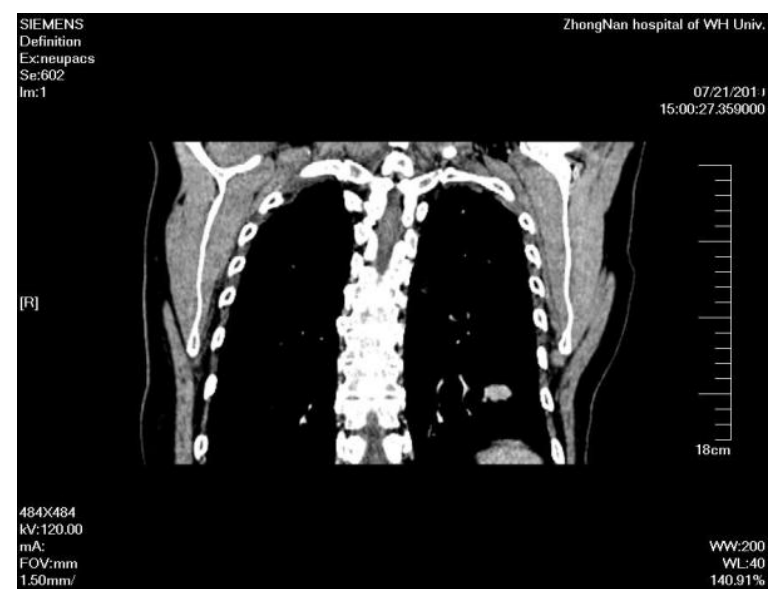

Fig 2c: Coronal MPR reconstruction demonstrates the nodule.

age of presentation in our case was 16 years which was higher to that of the reported literature. There seem to be no predilection to any particular sex though in our case there was male predominance which may not be significant due to the fewer number of cases.

Etiological mechanism of IMT is not well understood with several hypothesis proposed for the inflammatory changes attributable to a metabolic disturbance, organized cellular growth developing in association with pulmonary infection, viral origin or antigenantibody interaction in relation to an agent which is no longer identifiable. Although a history of antecedent infection can be attributable in almost a third of the cases, cultures of the resected specimens are unable to demonstrate growth of any organisms. ${ }^{2}$ 


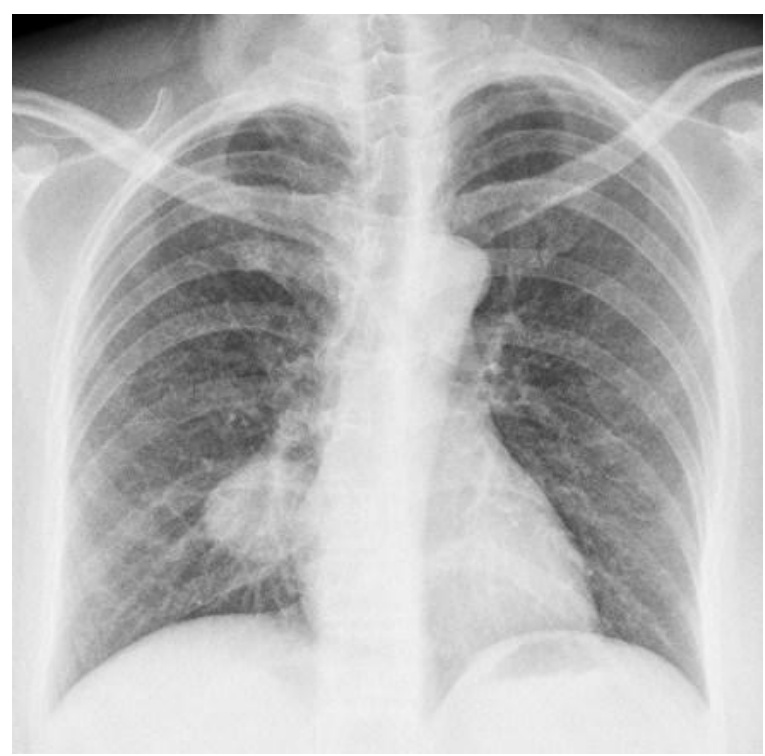

Fig 3a: Chest $X$-ray PA view demonstrates a round mass in the right lower lung field.

The condition is usually asymptomatic and identified incidentally in majority of the cases. Clinical symptoms may be related to location of the lesion as whether pulmonary, mediastinal or tracheobronchial region. Fourteen patients $(70 \%$ of all cases) in our study had no any clinical symptoms related to the lesion. In those that were symptomatic the most common presenting symptom was chest pain.

Histopathologically, inflammatory pseudotumor includes a spectrum of myofibroblastic proliferation with varying infiltrate of inflammatory cells. Based on the predominant histopathological component, myofibroblastic inflammatory tumor of the lung has been classified into three sub types: organizing pneumonia type, fibrous histiocytoma type and lymphoplasmacytic type with considerable overlap among the three subtypes. These tumors are thought to originate as organizing intraalveolar pneumonia. ${ }^{3}$ These tumors demonstrate vimentin, muscle specific actin and focally desmin within the cytoplasm of the spindle cells depicting myofibroblastic differentiation. Various conditions that must be recognized and differentiated includes malignant lymphoma, lymphoid hyperplasia, pseudolymphoma, plasmacytoma, malignant fibrous histiocytoma, sarcomatoid carcinoma of the lung, sclerosing hemangioma, sarcoma, and/or nodular chronic pneumonitis. ${ }^{4}$

Although disease has been labeled as benign, certain quasineoplastic features have led some to speculate the true nature of the lesion. Demonstration of clonal abnormalities, ALK and p80 as well as certain chromosomal rearrangement as $2 \mathrm{p} 23$ in IMT are suggestive of neoplasm further supplemented by evidence of recurrence or distant metastasis thus warranting complete surgical resection. Some reports of sarcomatous transformation of these pseudotumors have been reported in the literature.

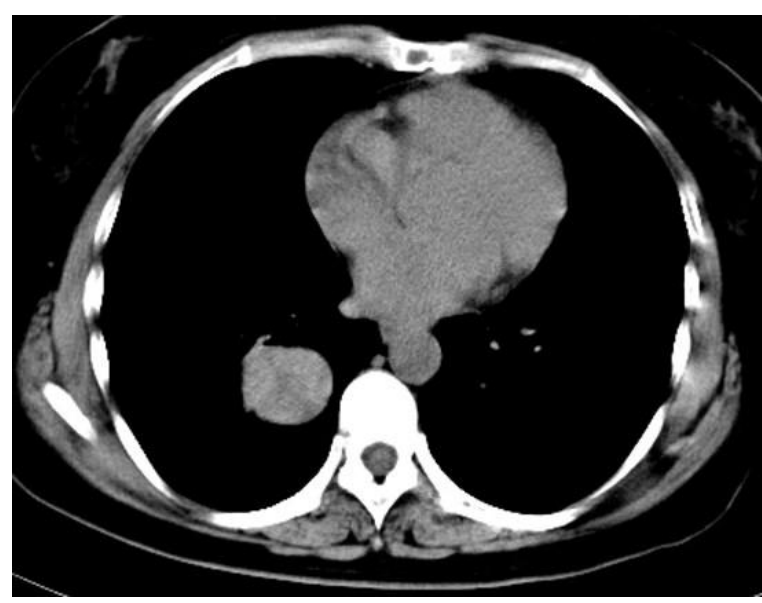

Fig 3b: Non-contrast $\mathrm{CT}$ in the same patient demonstrates the lesion.

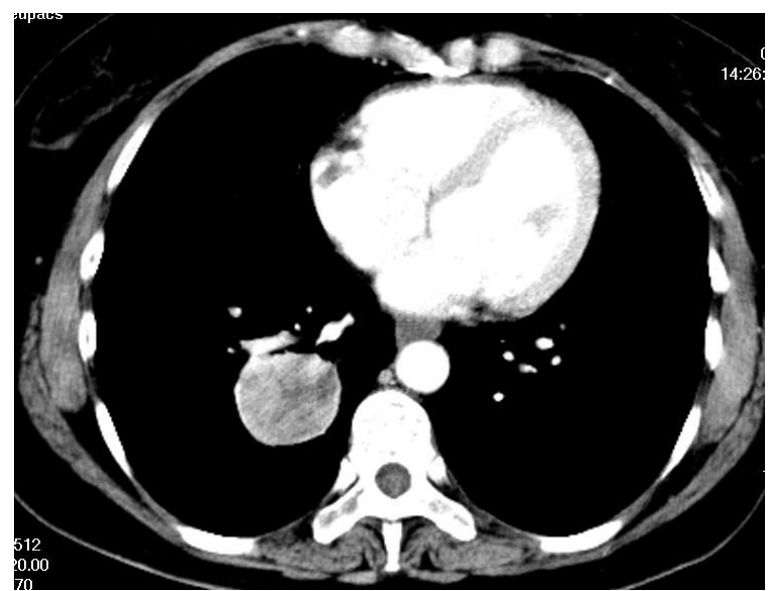

Fig 3c: Contrast enhanced CT scan demonstrates heterogeneous enhancement of the lesion. 
Radiologic features of inflammatory pseudotumors of the lung does not correlate with the histological patterns and are much varied and non specific thus posing diagnostic challenge while differentiating from other benign and malignant lesions. The most common radiographic presentation is the presence of a well circumscribed solitary mass which have more predilection to involve the right side particularly the lower lobes. ${ }^{5}$ Lesions are predominantly peripheral in location with central lesions relatively uncommon. Multiple involvement as in one of our case is rare and present in fewer than 5 $\%$ of cases. Calcification is more frequently seen in children than in adults. The various pattern of calcification observed in inflammatory pseudotumor of the lung ranges from an amorphous, mixed or fine fleck like pattern to densely mineralized. At times, calcification is difficult to discern radiographically while can be depicted on CT scan. ${ }^{6}$ Primary involvement of the mediastinum or hilar structures are not a common features, we observed only one case which had predominant lesion in the mediastinum and with extension into the peripheral lung. Endobronchial or endotracheal involvement may lead to segmental consolidation or atelectasis as in one of our case. ${ }^{7}$ Invasion to the mediastinum, hilum or presence of lymphadenopathy is relatively rare as is the presence of pleural effusion. In our case, abutting the pleural surface was noted in three cases. Increased parenchymal density around the lesion has been noticed in some studies which are related to peribronchial fibrosis with a patchy distribution, peribronchial lymphoplasmacytic infiltrates and intraalveolar organization and edema. A poorly circumscribed lesion with spiculated margins causes difficulty in differentiating from malignant lesions ${ }^{8,9}$ Aggressive features of inflammatory pseudotumor have been reported which include vertebral destruction, vascular invasion. ${ }^{8}$

CT aids in the delineation of the geographical location of the lesion besides added value in studying the internal characteristics of the lesions. In two of our cases, CT demonstrated central necrosis. CT by providing the anatomical details and lesions relation to surrounding structures or endotracheal, endobronchial extension helps in stratification of the management of such cases. CT guided biopsy is indispensable as a primary tool in the diagnosis of such cases. MRI features that have been described include a heterogeneous $\mathrm{T} 1$ signal which is slightly greater than that of skeletal muscle and also a characteristically high T2 signal. Lesions were seen to demonstrate diffuse heterogeneous enhancement on administration of gadolinium as contrast agent. Some studies have shown that inflammatory pseudotumor demonstrate high uptake of 18F-fluorodeoxyglucose (FDG) in FDG-positron emission tomography (PET).This finding in particular posed difficulty in differentiating from lung malignancies as carcinoma and sarcomas as well as other conditions including tuberculosis, fungal infection or rare entities like endometriosis ${ }^{10}$ that readily demonstrate vivid uptake. The radiological differential diagnosis of pseudotumor includes other causes of solitary pulmonary nodules or masses as primary neoplasm, metastasis, hamartoma, chondroma, sclerosing hemangioma, pulmonary granuloma. Inflammatory pseudotumor which are predominantly endobronchial usually need to be differentiated form carcinoid tumors.

Various schools of thought exist for the management of inflammatory pseudotumors recognizing its clinical complications or potential malignant transformation. Some advocate complete resection in all cases while others report complete remission by steroid or radiotherapy alone. Since the diagnostic value of needle aspiration or biopsy is questionable, resection in toto plays role not only in diagnosis but also has therapeutic benefit. Non-surgical treatment like radiotherapy and steroids have thus been reserved for the cases in the setting of incomplete surgical resection, tumor 


\begin{tabular}{|c|c|c|c|c|c|c|c|c|c|c|c|}
\hline \multicolumn{12}{|c|}{ Table 1: CT characteristics of the 20 cases of inflammatory myofibroblastic tumor of lungs } \\
\hline Case & $\begin{array}{l}\text { Age in } \\
\text { yrs) / } \\
\text { Sex }\end{array}$ & $\begin{array}{l}\text { Clinical } \\
\text { features }\end{array}$ & Site & $\begin{array}{l}\text { Predominant } \\
\text { location }\end{array}$ & Pattern & $\begin{array}{l}\text { Dimension } \\
\text { (maximum } \\
\text { in cm) }\end{array}$ & Shape & $\begin{array}{l}\text { Defined } \\
\text { Edge }\end{array}$ & $\begin{array}{l}\text { Atypical } \\
\text { findings }\end{array}$ & Attenuation & $\begin{array}{l}\text { Degree of } \\
\text { enhancem- } \\
\text { ent }\end{array}$ \\
\hline 1 & 32/M & Asymptomatic & LLL & Parenchymal & SPN & 2.8 & Round & Well & Abuts pleura & Homogeneous & Marked \\
\hline 2 & $46 / \mathrm{F}$ & Cough & $\begin{array}{l}\text { RUL/ } \\
\text { RML }\end{array}$ & Parenchymal & Mass & 6.2 & $\begin{array}{l}\text { Wedge } \\
\text { shaped }\end{array}$ & Poorly & Abuts pleura & Heterogeneous & Moderate \\
\hline 3 & 37/M & Chest pain & RUL & Parenchymal & SPN & 2.3 & Round & Well & None & Homogeneous & Mild \\
\hline 4 & 53/M & Chest pain & RUL & Parenchymal & SPN & 1.8 & Round & Well & None & Homogeneous & Mild \\
\hline 5 & $27 / \mathrm{F}$ & Asymptomatic & LLL & Parenchymal & SPN & 1.3 & Round & Well & None & Homogeneous & Mild \\
\hline 6 & 29/M & Asymptomatic & RUL & Parenchymal & MPN & $2.9 / 1.8$ & Round & Well & None & Homogeneous & Mild \\
\hline 7 & $43 / \mathrm{M}$ & Asymptomatic & RLL & Parenchymal & Mass & 4.9 & Round & Well & None & Homogeneous & Mild \\
\hline 8 & 29/M & Asymptomatic & RUL & Parenchymal & SPN & 2.6 & Oval & Well & None & Homogeneous & Mild \\
\hline 9 & 16/M & Asymptomatic & RUL & $\begin{array}{l}\text { Mediastinal/ } \\
\text { Parenchymal }\end{array}$ & Mass & $11 . .2$ & Round & Poorly & $\begin{array}{l}\text { Necrosis, } \\
\text { abuts pleura }\end{array}$ & Heterogeneous & Mild \\
\hline 10 & $26 / \mathrm{M}$ & Asymptomatic & RUL & Parenchymal & SPN & 1.8 & Round & Well & None & Homogeneous & Mild \\
\hline 11 & $32 / \mathrm{F}$ & Asymptomatic & RUL & Parenchymal & SPN & 2.3 & Round & Well & None & Homogeneous & Mild \\
\hline 12 & $48 / \mathrm{F}$ & Asymptomatic & RUL & Parenchymal & SPN & 1.6 & Round & Well & None & Homogeneous & Mild \\
\hline 13 & $61 / \mathrm{M}$ & $\begin{array}{l}\text { Fever, weight } \\
\text { loss }\end{array}$ & RUL & Parenchymal & Mass & 5.2 & Lobulated & Poorly & None & Heterogeneous & Mild \\
\hline 14 & 39/M & Asymptomatic & RUL & Parenchymal & SPN & 1.8 & Round & Well & None & Homogeneous & Mild \\
\hline 15 & $42 / \mathrm{F}$ & Asymptomatic & RUL & Parenchymal & SPN & 1.1 & Round & Well & None & Homogeneous & Moderate \\
\hline 16 & $37 / \mathrm{M}$ & Asymptomatic & $\begin{array}{l}\text { RUL/ } \\
\text { RML }\end{array}$ & Parenchymal & MPN & $2.0 / 1.3$ & Round & Well & None & Homogeneous & Mild \\
\hline 17 & $36 / \mathrm{F}$ & Chest pain & LLL & Parenchymal & Mass & 10.5 & Lobulated & Well & Calcification & Heterogeneous & Mild \\
\hline 18 & 59/M & Asymptomatic & RUL & Parenchymal & SPN & 2.4 & Round & Well & None & Homogeneous & Mild \\
\hline 19 & $34 / \mathrm{F}$ & Asymptomatic & RUL & Parenchymal & SPN & 2.7 & Round & Well & None & Homogeneous & Mild \\
\hline 20 & $52 / \mathrm{M}$ & Hemoptysis & RML & Endobronchial & $\begin{array}{l}\text { Mass } \\
\text { Conso- } \\
\text { lidation }\end{array}$ & 9.2 & Lobulated & Well & Necrosis & Heterogeneous & Marked \\
\hline
\end{tabular}


recurrence or when patient is unable to undergo surgery. ${ }^{11}$

To conclude, inflammatory pseudotumor poses a diagnostic challenge both clinically and radiologically with histopathological diagnosis mandatory in almost all cases. In equivocal lung lesions presenting as solitary nodules or masses especially in pediatric population, inflammatory pseudotumors should be kept in as a differential diagnosis.

\section{References}

1. Rossi SE, McAdams HP, Erasmus JJ, Sporn TA. A 63-year-old woman with a 2month history of dyspnea. Chest 2000; 117:1505-1507.

2. Singh RS, Dhaliwal RS, Puri D, Behera D, Das A. Inflammatory pseudotumour of the lung: report of a case and review of literature. Indian $\mathrm{J}$ Chest Dis Allied Sci 2001;43:231-234.

3. Matsubara O, Tan-Liu NS, Kenney RM, Mark EJ. Inflammatory pseudotumors of the lung: progression from organizing pneumonia to fibrous histiocytoma or to plasma cell granuloma in 32 cases. Hum Pathol 1988;19:807-814.

4. Sakurai H, Hasegawa T, Watanabe S, Suzuki K, Asamura H, Tsuchiya R. Inflammatory myofibroblastic tumor of the lung. Eur J Cardiothorac Surg 2004; 25:155-159.

5. Agrons GA, Rosado-de-Christenson ML, Kirejczyk WM, Conran RM, Stocker JT. Pulmonary inflammatory pseudotumor: radiologic features. Radiology 1998;206 :511-518.
6. Patel S, Shah D, Goswami K, Shah S, Shah P. Inflammatory pseudotumor of lung, a case report and review of literature. Indian Journal of Radiology and Imaging 2006;16:117-120.

7. Shapiro MP, Gale ME, Carter BL. Variable CT appearance of plasma cell granuloma of the lung. J Comput Assist Tomogr 1987;11:49-51.

8. Hedlund GL, Navoy JF, Galliani CA, Johnson WH, Jr. Aggressive manifestations of inflammatory pulmonary pseudotumor in children. Pediatr Radiol 1999;29:112-116.

9. Kakitsubata Y, Theodorou SJ, Theodorou DJ, Nabeshima K, Kakitsubata S, Friedman PJ. Myofibroblastic inflammatory tumor of the lung: CT findings with pathologic correlation. Comput Med Imaging Graph 2007; 31:607-613.

10. Huellner MW, Schwizer B, Burger Iet al. inflammatory pseudotumor of the lung with high FDG uptake. Clin Nucl Med 2010;35:722-723.

11. Cerfolio RJ, Matthews TC. Resection of the entire left mainstem bronchus for an inflammatory pseudotumor. Ann Thorac Surg 2005;79:2127-2128. 